\title{
Integrative Analysis of microRNA, mRNA and aCGH Data Reveals Asbestos- and Histology-Related Changes in Lung Cancer
}

\author{
Penny Nymark, ${ }^{1,2 \dagger}$ Mohamed Guled, ${ }^{1 \dagger}$ Ioana Borze, ' Ali Faisal, ${ }^{3}$ Leo Lahti, ${ }^{2,3}$ Kaisa Salmenkivi, \\ Eeva Kettunen, ${ }^{2}$ Sisko Anttila, ${ }^{1,2}$ and Sakari Knuutila* \\ 'Department of Pathology, Haartman Institute and HUSLAB, University of Helsinki and Helsinki \\ University Central Hospital, Helsinki, Finland \\ ${ }^{2}$ Biological Mechanisms and Prevention of Work-related Diseases, Health and Work Ability, \\ Finnish Institute of Occupational Health, Helsinki, Finland \\ ${ }^{3}$ Department of Information and Computer Science, Aalto University, Aalto, Finland
}

Lung cancer has the highest mortality rate of all of the cancers in the world and asbestos-related lung cancer is one of the leading occupational cancers. The identification of asbestos-related molecular changes has long been a topic of increasing research interest. The aim of this study was to identify novel asbestos-related molecular correlates by integrating miRNA expression profiling with previously obtained profiling data (aCGH and mRNA expression) from the same patient material. miRNA profiling was performed on 26 tumor and corresponding normal lung tissue samples from highly asbestos-exposed and non-exposed patients, and on eight control lung tissue samples. Data analyses on miRNA expression, and integration of miRNA and previously obtained mRNA data were performed using Chipster. A separate analysis was used to integrate miRNA and previously obtained aCGH data. Both known and new lung cancer-associated miRNAs and target genes with inverse correlation were discovered. Furthermore, DNA copy number alterations (e.g., gain at I2p|3.3I) were correlated with the deregulated miRNAs. Specifically, thirteen novel asbestos-related miRNAs (over-expressed: miR-|48b, miR-374a, miR-24-I*, Let-7d, Let-7e, miR-199b-5p, miR-33I-3p, and miR-96 and under-expressed: miR-939, miR-67I-5p, miR-605, miR-I224-5p and miR-202) and inversely correlated target genes (e.g., GADD45A, LTBPI, FOSB, NCALD, CACNA2D2, MTSSI, EPB4 IL3) were identified. In addition, over-expression of the well known squamous cell carcinoma-associated miR-205 was linked to down-regulation of the DOK4 gene. The miRNAs/genes presented here may represent interesting targets for further investigation and could eventually have potential diagnostic implications. (c) 20II Wiley-Liss, Inc.

\section{INTRODUCTION}

Lung cancer is the leading cause of cancer death throughout the world. Although the major risk factor for lung cancer is tobacco smoke, an estimated $5-7 \%$ of all these cancers are attributable to asbestos exposure (LaDou, 2004). Asbestos-related lung cancer is considered to be one of the most important occupational cancers (Karjalainen et al., 1994).

Asbestos is the common commercial term for six naturally occurring silicate mineral fibers. The material has been widely used in building material (LaDou, 2004). Asbestos fibers can induce different types of alterations in normal cell homeostasis, such as oxidative stress, inflammation, DNA damage, mitochondrial dysfunctions, and apoptosis (Nymark et al., 2008). In combination with tobacco smoke, asbestos exposure increases the risk of lung cancer in a synergistic manner, which has made it exceedingly difficult to elucidate those alterations which are related specifically to asbestos exposure, since many asbestos workers are also tobacco smokers (Vainio and Boffetta, 1994). We have previously identified 18 chromosomal regions harboring asbestos-related copy number alterations (CNA) using array $\mathrm{CGH}$ $(\mathrm{aCGH})$ and six of these regions also showed asbestos-related gene expression (mRNA) changes (Nymark et al., 2006; Wikman et al., 2007). Three of the CNAs, at 2p16, 9q33.1 and

\footnotetext{
Additional Supporting Information may be found in the online version of this article.

†Penny Nymark and Mohamed Guled contributed equally to this work.

Supported by: The Academy of Finland, Grant number: 115372; Finnish Funding Agency for Technology and Innovation (TEKES), Grant number: 40101/07; Maud Kuistila Memorial Foundation; the Swedish Cultural Foundation in Finland; the Ida Montin Foundation; the Jalmari and Rauha Ahokas foundation; the K. Albin Johansson Foundation; Helsinki Institute for Information Technology (HIIT) and the Finnish Center of Excellence on Adaptive Informatics Research (AIRC).

*Correspondence to: Sakari Knuutila, Haartmaninkatu 3, 00014

Helsingin Yliopisto. E-mail: sakari.knuutila@helsinki.fi

Received 28 December 2010; Accepted 23 March 2011

DOI 10.1002/gcc.20880

Published online in

Wiley Online Library (wileyonlinelibrary.com).
} 
19p13, were studied using other methods and were confirmed to be asbestos-related in a larger study population (Ruosaari et al., 2008; Kettunen et al., 2009; Nymark et al., 2009). In this study, we have profiled the miRNAome of the same samples and integrated the data with earlier mRNA and aCGH results.

MicroRNAs (miRNAs) have rapidly become an attractive method for profiling cancers. These noncoding small RNAs of about 19-25 nucleotides have proved to be more efficient in distinguishing between tumor histology, classifying undifferentiated tumors and predicting patient outcome compared with traditional gene expression profiling of mRNAs (Wang et al., 2009). Their role in asbestos-related lung cancer has not been investigated previously.

\section{MATERIALS AND METHODS}

\section{Samples}

The miRNAome of 13 malignant lung tumors and corresponding normal lung samples from highly asbestos-exposed patients as well as 13 tumors and corresponding normal samples from nonexposed patients was analyzed. Throughout the text "normal" will refer to the normal samples from the cancer patients. The two groups were matched for age, gender (male), nationality (Finnish), smoking history and distribution of histological types (Table 1). The samples were obtained during lung cancer surgery at the Helsinki University Central Hospital in the 1990s. All patients were personally interviewed for their smoking and work history. Informed consent to take part in the study and to use their tissue was obtained. The study protocols were approved by the Ethical Review Board for Research in Occupational Health and Safety and the Coordinating Ethical Review Board, Helsinki and Uusimaa Hospital District (75/E2/2001).

Asbestos fiber counts were measured by electron microscopy with energy dispersive spectrometry of lung tissue (Karjalainen et al., 1993). The exposed group consisted of patients with a definite or probable exposure according to work history and a pulmonary asbestos fiber count higher than 5 million fibers/g of dry lung tissue (mainly amphiboles). An asbestos fiber concentration of 2 to 5 million is thought to confer roughly a twofold increase in the risk of lung cancer (Karjalainen et al., 1994; Consensus Report, 1997). The nonexposed group consisted of patients with no known asbestos exposure according to their work history and with an asbestos fiber count of 0 million fibers/g of dry lung tissue or in one case 0.5 million fibers $/ g$. It has been reported that up to 1 million fibers/g of dry lung tissue can be detected in the general population (Churg, 1997).

The miRNAome was also analyzed in eight control samples, of which seven were lung tissue samples from patients without cancer. Two of the patients had been exposed to asbestos according to the work history and had pulmonary asbestos fiber counts of 2.8 and 3.0. Two patients had less than 1 million fibers and no asbestos exposure according to the work history. Three patients had no known history of asbestos exposure, but the fiber count had not been determined. All seven patients had a history of smoking (Table 1). The eighth sample was a commercially available lung tissue RNA sample (FirstChoice ${ }^{\circledR}$ Human total RNA, Applied Biosystems/Ambion, USA).

mRNA data were available from all (Table 1) tumor samples and from 22 of the 26 normal samples, as well as from four of the control samples (Wikman et al., 2007). aCGH data were available from 18 of the 26 tumor samples (Nymark et al., 2006).

\section{RNA Extraction}

Total RNA extraction was performed by using the miRNeasy Mini Kit (Qiagen, Valencia, CA) according to the manufacturer's protocol. The total RNA samples were stored at $-70^{\circ} \mathrm{C}$. NanoDrop ND-1000 Spectrophotometer (NanoDrop Technologies, Wilmington, Delaware, USA) was used for the quantification of the eluted RNA. The quality and the quantity of miRNAs were assessed with the Agilent 2100 Bioanalyzer using RNA Nano 6000 and Small RNA Chips, according to the manufacturer's protocol (Agilent Technologies, Palo Alto, CA).

\section{miRNA Microarrays}

Agilent's miRNA Complete Labeling and Hybridization Kit V2 (Protocol Version 2.1, Agilent Technologies, Palo Alto, CA) was used for processing the samples. Briefly, $100 \mathrm{ng}$ of total RNA was dephosphorylated and labeled with Cyanine-3-pCp. The samples were dried in a vacuum concentrator at medium-high heat setting, resuspended in nuclease-free water and hybridized on the Agilent Human miRNA v2 (723 human and 76 human viral miRNAs, Sanger database v.10.1). A high-resolution Microarray Scanner (G2565CA, Agilent Technologies, Palo Alto, 
TABLE I. Patients and Controls

\begin{tabular}{|c|c|c|c|c|c|c|}
\hline \multirow[b]{2}{*}{ Sample no. ${ }^{a}$} & \multirow[b]{2}{*}{ Age } & \multirow{2}{*}{$\begin{array}{l}\text { Asbestos } \\
\text { fiber count }{ }^{b}\end{array}$} & \multicolumn{3}{|c|}{ Smoking } & \multirow[b]{2}{*}{ Diagnosis } \\
\hline & & & Mean cig/day & $P K Y^{c}$ & current/ex & \\
\hline \multicolumn{7}{|c|}{ Exposed patients } \\
\hline I & 64 & 72.9 & 20 & 33 & ex & $A C$ \\
\hline 2 & 59 & 12.6 & 50 & 105 & cur & $A C$ \\
\hline 3 & 59 & 35.0 & 20 & 36 & ex & $A C$ \\
\hline 4 & 65 & 9.4 & 10 & 25 & cur & $A C$ \\
\hline 5 & 65 & 10.8 & 15 & 23 & ex & $A C$ \\
\hline 7 & 62 & 6.0 & 30 & 65 & ex & SCC \\
\hline 8 & 65 & 5.9 & 20 & 32 & cur & SCC \\
\hline 9 & 57 & 6.6 & 20 & 36 & ex & SCC \\
\hline 10 & 67 & 8.4 & 20 & 20 & ex & LCLC \\
\hline II & 66 & 19.0 & 15 & 35 & ex & LCLC \\
\hline 12 & 58 & 90 & 30 & 65 & cur & LCLC \\
\hline 13 & 64 & 145 & 20 & 22 & ex & $\mathrm{AC} / \mathrm{SCC}$ \\
\hline 14 & 62 & 12.8 & 23 & 55 & cur & SCLC \\
\hline mean & 62.5 & 33.4 & 23 & 42.5 & $38 \%$ cur & $38 \%$ AC \\
\hline \multicolumn{7}{|c|}{ Nonexposed patients } \\
\hline 15 & 55 & 0.0 & 20 & 36 & cur & $A C$ \\
\hline 16 & 69 & 0.0 & 20 & 47 & cur & $A C$ \\
\hline 17 & 70 & 0.0 & 15 & 38 & ex & $A C$ \\
\hline 18 & 65 & 0.0 & 20 & 52 & cur & $A C$ \\
\hline 20 & 67 & 0.0 & 25 & 50 & cur & $A C$ \\
\hline 21 & 65 & 0.0 & 20 & 47 & ex & SCC \\
\hline 22 & 65 & 0.0 & 15 & 25 & ex & SCC \\
\hline 23 & 50 & 0.0 & 20 & 35 & cur & SCC \\
\hline 24 & 64 & 0.0 & 20 & 45 & cur & SCC \\
\hline 25 & 67 & 0.0 & 20 & 47 & ex & SCC \\
\hline 27 & 72 & 0.5 & 22 & 36 & ex & LCLC \\
\hline 28 & $4 I$ & 0.0 & 25 & 31 & ex & $\mathrm{AC} / \mathrm{SCC}$ \\
\hline 29 & 64 & 0.0 & 30 & 66 & cur & SCLC \\
\hline \multirow{2}{*}{\multicolumn{7}{|c|}{ Control patients ${ }^{d}$}} \\
\hline & & & & & & \\
\hline 30 & - & - & - & - & - & Commercial lung RNA \\
\hline 31 & 51 & 0.0 & - & - & cur & Intrapulmonary lymph node $\mathrm{e}^{\mathrm{e}}$ \\
\hline 32 & 68 & 0.0 & 2 & - & cur & Hamartoma $\mathrm{e}^{\mathrm{e}}$ \\
\hline 33 & 32 & 0.0 & - & - & ex & Hamartoma $^{\mathrm{e}}$ \\
\hline 34 & 67 & $<0.3$ & 10 & 23 & ex & Lymphoid hyperplasia $^{\mathrm{e}}$ \\
\hline 35 & 55 & 0.9 & 20 & 46 & cur & Tuberculoma ${ }^{\mathrm{e}}$ \\
\hline 36 & 56 & 2.8 & 30 & 48 & cur & Inflammatory pseudotumour \\
\hline 37 & 68 & 3.0 & 15 & 31 & ex & Lung abscess $\mathrm{e}^{\mathrm{e}}$ \\
\hline mean & & 0.7 & 18 & 37 & $57 \%$ cur & \\
\hline
\end{tabular}

LCLC, large cell lung cancer; AC/SCC, adeno-squamous cell carcinoma; SCLC, small cell lung cancer.

a Same samples as in (Nymark et al., 2006).

billion fibers/g dry lung tissue, detection limit $=<0$.I.

cPack years (20 cigarettes/day).

'Patients without cancer.

e Control samples taken outside the pathological area.

CA) was used to scan the microarrays and the obtained images were processed with Agilent Feature Extraction Software v. 9.5.

\section{Data Analysis}

miRNA data

The miRNA data (GEO accession: GSE25508) were analyzed using Chipster version 1.4.2 (http://chipster.csc.fi/). The data were normalized according to the default settings in Chipster for Agilent miRNA(v2) platform. The $\log _{2}$ transformed data were preprocessed using the coefficient of variation (CV-filter) to filter out miRNAs with no variation across the patients. The miRNAs with $20 \%$ or $50 \%$ (setting 0.8 or 0.5 depending on the analysis and number of samples) largest variation were chosen for further analysis. Statistical analyses using principal components analysis (PCA) and two-group tests were then 
performed on the normalized, preprocessed data to obtain differentially expressed miRNAs with adjusted $P$ values below 0.05 or 0.2 , depending on the analysis. The $P$ values were adjusted by multiple testing using default Chipster settings (Benjamini-Hochberg test) and thus, indicate false discovery rate (FDR). For example, an adjusted $P$ value of 0.1 indicates that the expected fraction of false positive findings is $10 \%$.

\section{Integrative analysis on miRNA and mRNA data}

The mRNA data were also $\log _{2}$ transformed and analyzed in Chipster similarly to the miRNA data. Correlations between the miRNA and mRNA data were analyzed using the "Up-down analysis of miRNA targets"-tool (default settings, i.e., target genes were fetched from targetScan and PicTar). The significantly differentially expressed miRNAs obtained in group comparisons were tested with the analysis tool on significantly differentially expressed mRNAs obtained in group comparisons (two-group test on $90 \% \mathrm{CV}$ filtered, with expression values $>50$ in at least $50 \%$ of the samples) or on preprocessed $(90 \% \mathrm{CV}$ filtered, expression value $>50$ in at least $50 \%$ of the samples) mRNA data. Slight nonsignificant differences in expression of an mRNA between the groups can be considered as significant if the targeting miRNA shows significant opposite expressional differences between the groups.

\section{Integrative analysis on miRNA and aCGH data}

An integrated analysis was performed on the DNA copy number (aCGH) and miRNA data to detect co-ordinated patterns in various patient groupings. A common pooled reference was used for all of the aCGH hybridizations. To achieve a similar setting for miRNAs, the independent control patients' samples were pooled and used as a reference. To characterize differential expression, quantile normalization was performed based on the results from Pradervand et al., (2009) and Lopez-Romero et al., (2010). The aCGH was measured from Agilent human cDNA array version 1.0 arrays, where the probes were mapped according to human genome build GRCh37 using annotations from CanGEM. Circular binary segmentation was used to translate noisy measurements into regions of equal copy number, using the DNA copy number data analysis in R package version 1.22.0 (Venkatraman and Olshen, 2007). The largest plateaus of the chromosome were used as a means to call segments of gain and loss. Thus, three levels were obtained for both miRNA and aCGH; over-expression/gain, under-expression/loss and no change. Finally the dataset was made comeasurable by mapping aCGH regions to the corresponding genomic location for the miRNAs in each patient.

This analysis was performed on a set of eight different groups of patients; (i) all patients, (ii) asbestos exposed, (iii) nonasbestos exposed, (iv) adenocarcinoma (AC) samples, (v) asbestosexposed patients' AC samples, (vi) squamous cell carcinoma (SCC) samples, (vii) asbestos-exposed patients' SCG samples, (viii) cancer groups 1 and 2 (described in the Results section, "Lung cancer-related miRNAs").

For each miRNA, a Spearman rank correlation was computed between the two profiles (copy number $[\mathrm{CN}]$ and miRNA) across the patients for the group. Empirical $P$ values were calculated by permuting the whole $\mathrm{CN}$ and miRNA matrix of the group such that the distribution of miRNAs and $\mathrm{CN}$ remained the same but any relationships were removed.

The integrative analysis was confirmed with the "pint" package of R/BioConductor (http:// www.bioconductor.org/packages/devel/bioc/html/ pint.html) that has been designed for data integration tasks in functional genomics (Lahti et al., 2009).

\section{RESULTS}

\section{Lung Cancer-Related miRNAs}

Thirty-four miRNAs were identified to be significantly differentially expressed (FDR $<0.05)$ in tumor samples compared to their normal counterparts (Fig. 1, Supporting Information 1). The 34 miRNAs classified the normal samples into one group and the tumor samples into two separate groups, one distantly and one closely related to the normal samples. In the following sections we will refer to these groups as cancer Groups 1 and 2 (Fig. 1). The frequency of samples with AC histology was significantly higher in Group 1 (8/ $13,62 \%)$ than in Group $2(2 / 13,15 \%, P<0.05$; Table 2).

Integrated analysis with 771 differentially expressed mRNA probes $(\mathrm{FDR}<0.05)$ revealed 27 down-regulated target genes correlating with 10 of the over-expressed miRNAs and 33 upregulated target genes correlating with five of the under-expressed miRNAs (Supporting Information 2 and 3 ). 


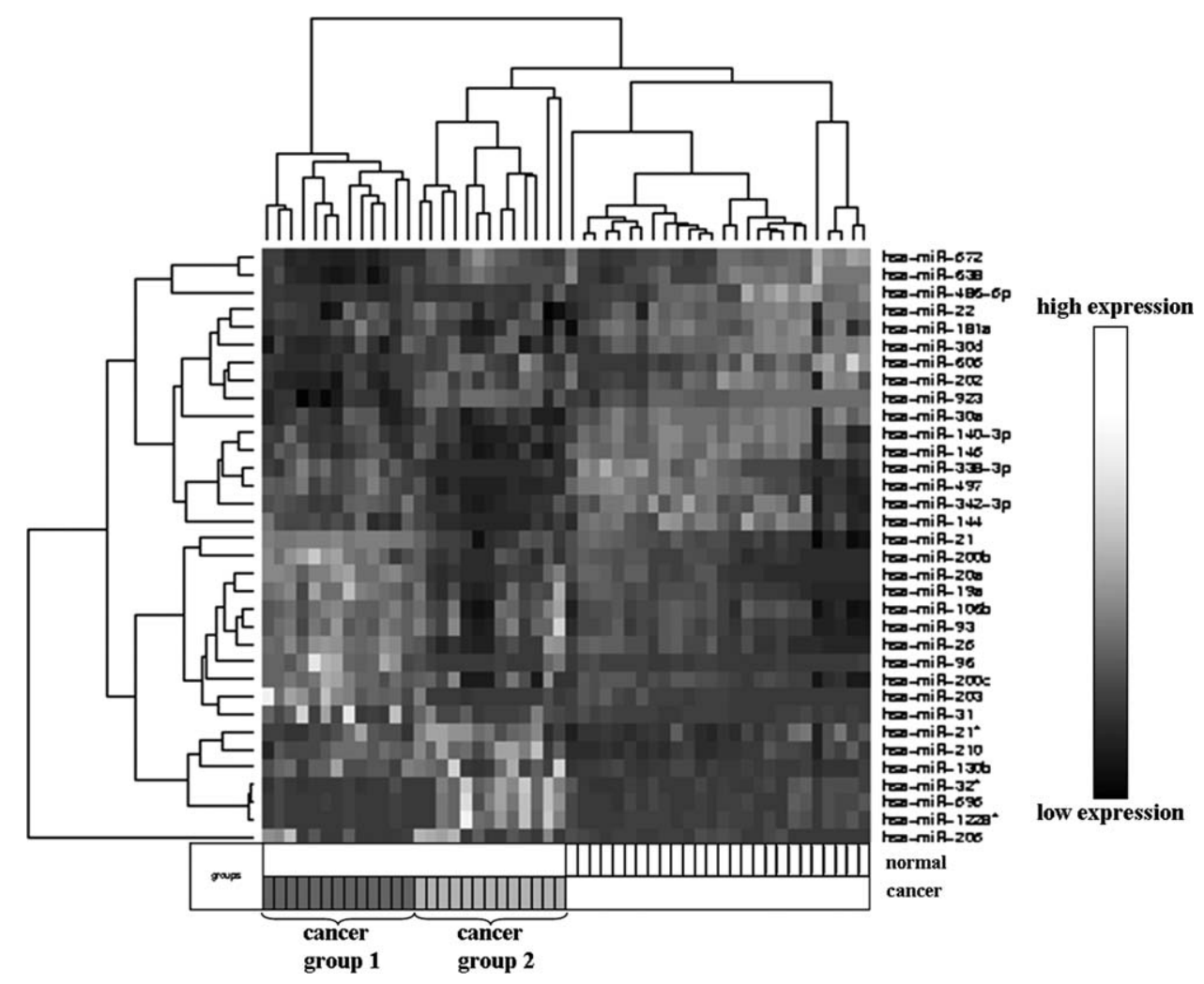

Figure I. Thirty-four significantly differentially expressed miRNAs (FDR $<0.05)$ in tumor compared with normal samples.

TABLE 2. Characteristics of Cancer Groups I and 2 Identified in the miRNA Profiling Analysis Comparing Tumour and Normal Samples (Fig. I)

\begin{tabular}{lcccccc}
\hline & & & \multicolumn{3}{c}{ Smoking } & \multicolumn{2}{c}{ Diagnosis } \\
\cline { 3 - 7 } & Mean age & Asbestos & exposure & Mean cig/day & Mean PKY & Current/ex \\
\hline Cancer groupl $(n=13)$ & 61.7 & $54 \%$ exposed & 21.9 & 43.8 & $38 \%$ cur & $62 \%$ AC \\
Cancer group2 $(n=13)$ & 63.5 & $46 \%$ exposed & 21.5 & 41.3 & $54 \%$ cur & $15 \%$ AC \\
P-value & 0.55 & 0.50 & 0.90 & 0.73 & 0.35 & 0.04 \\
\hline
\end{tabular}

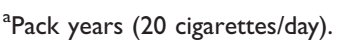

Integrated analysis on miRNA and aCGH data revealed six miRNAs affected by CNA in addition to being deregulated and four were confirmed by the "pint" package approach (see Methods). miR-200c (corr. $=0.32, \mathrm{FDR}=$ $0.0008)$ and miR-141 (corr. $=0.25, \mathrm{FDR}=0.001)$ at $12 \mathrm{p} 13.31$ were over-expressed and gained, while miR-27a at $19 \mathrm{p} 13.13$ (corr. $=0.22, \mathrm{FDR}=$ 0.0016 ) and miR-205 at $1 \mathrm{q} 32.2$ (corr. $=-0.13$, $\mathrm{FDR}=0.0051)$ showed both types of correlating directions.

Sixty-eight miRNAs were differentially expressed between cancer Groups 1 and 2 (FDR $<0.05$, Supporting Information 4). Integrated analysis with aCGH data identified two of the 68 miRNAs to be affected by CNA in correlation with the deregulated expression. The first, miR-27a (19p13.1), was more frequently altered in cancer Group 1 and the second, miR-939 (8q24.3), in cancer Group 2. The two miRNAs alone were able to classify $96 \%$ (25/26) of the samples correctly (data not shown). Integrated analysis with preprocessed mRNA data revealed 15 target genes displaying an inverse correlation with the over-expression of miR-27a (Supporting Information 5). The most strongly affected was SYT1 (Fig. 2a).

\section{miRNA Expression in Normal Samples Compared with Controls}

Normal lung samples from lung cancer patients were compared with control samples to identify early carcinogenesis-related changes. Using the 

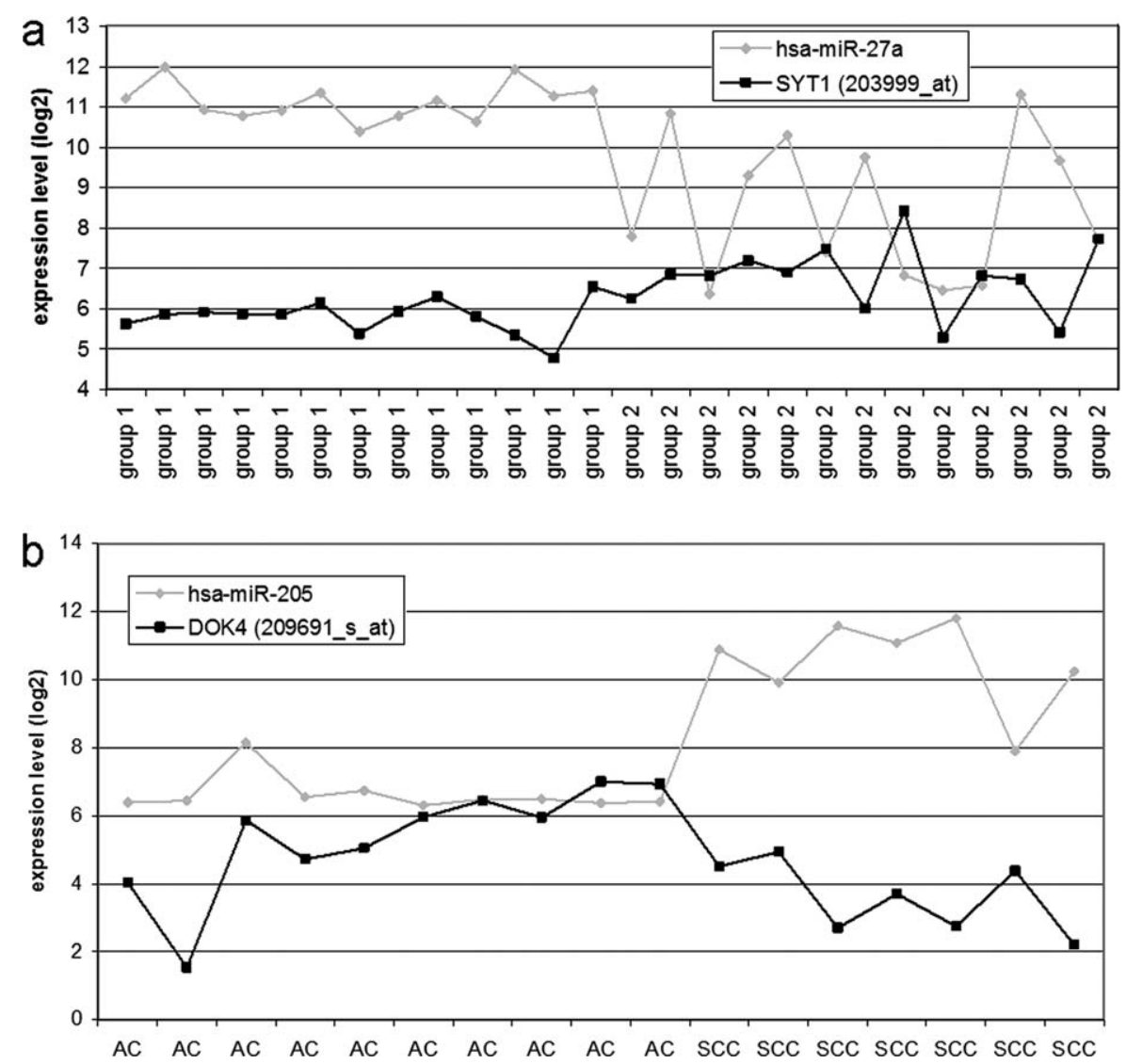

Figure 2. a: Expression of miR-27a and the target gene SYTI in cancer Groups I and 2. The median $\log 2$ fold change between cancer Groups I and 2 is 3.37 for miR-27a and -0.97 for SYTI. b: Expression of miR-205 and the target gene DOK4 in AC and SCC samples. The median log2 fold change between $A C$ and SCC is 4.42 for miR-205 and -1.78 for DOK4.

statistical PCA test in Chipster, one of the controls (sample nr. 34, Table 1) clustered together with the normal samples, while the others clustered separately, and was omitted from the analysis. One hundred miRNAs were significantly differentially expressed (FDR $<0.05$, data not shown). Further analysis was performed on the top 30 miRNAs (FDR $<0.0001$ ), of which $77 \%$ (23/30) were under-expressed in the normal samples compared with the controls (Supporting Information 6).

Integrated analysis with preprocessed mRNA data revealed 62 up-regulated target genes correlating with 15 of the under-expressed miRNAs (Supporting Information 7).

\section{Histology-Related miRNAs}

A comparison between AC and SCC samples revealed the previously known and highly SCCspecific miR-205 $\left(\right.$ FDR $=0.000005, \log _{2}$ fold change $=3.84$, predictive $\log _{2}$ expression level $\geq 7.89$, predictive value $94 \%$ ). Integrated analysis with 124 significantly differentially expressed
mRNAs between AC and SCG $(\mathrm{FDR}<0.25$; Supporting Information 8), detected inverse correlation of the target gene DOK4 (Fig. 2b).

\section{Asbestos-Related miRNAs}

Cancer Groups 1 and 2 (Fig. 1) were analyzed separately using PCA analysis. Cancer group 1 exhibited significant differences between the asbestos-related and nonrelated samples, while cancer Group 2 showed slight differences between current and ex-smokers (data not shown). Eight miRNAs (FDR $<0.05)$ distinguished asbestos-exposed from nonexposed patients' samples in Group 1 and were able to classify all of the samples correctly (Fig. 3a and Table 3). Integrated analysis with preprocessed mRNA data revealed three down-regulated target genes (GADD45A, LTBP1 and FOSB) correlating with the over-expression of miR-148b (Table 4). In Group 2, three miRNAs (FDR $<0.2)$ were differentially expressed between current and exsmokers; however, only 10/13 samples were classified correctly (Table 3). No inversely correlating 

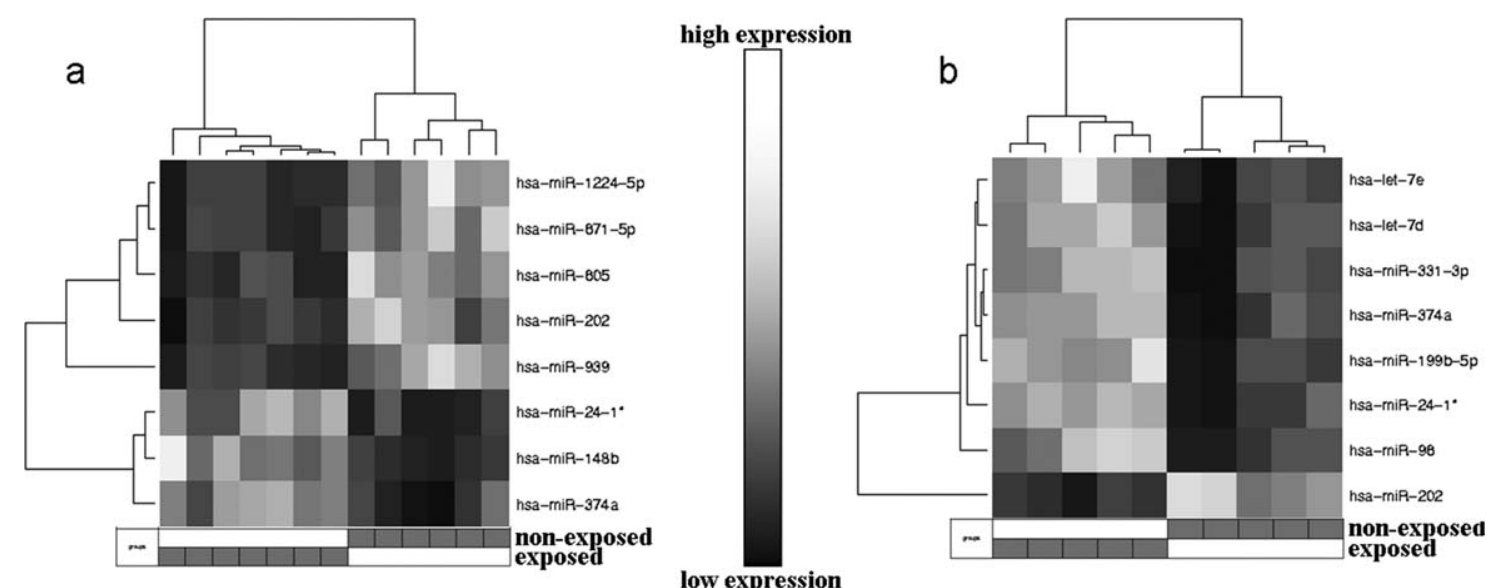

Figure 3. a: Eight differentially expressed miRNAs between asbestos-related and nonrelated tumor samples in cancer Group I. b: Eight differentially expressed miRNAs between asbestos-related and nonrelated $\mathrm{AC}$ samples.

TABLE 3. Asbestos- and Smoking Status-Related miRNAs Identified in Cancer Groups I and 2, and in AC Samples

\begin{tabular}{|c|c|c|c|c|}
\hline miRNA & $\log _{2}$ fold change ${ }^{a}$ & FDR & $\begin{array}{l}\text { Predictive } \log _{2} \\
\text { expression level }\end{array}$ & $\begin{array}{l}\text { Predictive } \\
\text { value }\end{array}$ \\
\hline \multicolumn{5}{|c|}{ Cancer group I } \\
\hline \multicolumn{5}{|c|}{ Over-expressed in asbestos-related samples } \\
\hline miR-I $48 b$ & 0.325 & 0.028543 & $\geq 6.73$ & $100 \%$ \\
\hline miR-374a & $0.53357 \mid 4$ & 0.028543 & $\geq 7.16$ & $76 \%$ \\
\hline $\operatorname{miR}-24-I^{*}$ & 0.19904763 & 0.047273 & $\geq 6.54$ & $76 \%$ \\
\hline \multicolumn{5}{|c|}{ Under-expressed in asbestos-related samples } \\
\hline miR-939 & -1.2571429 & 0.018745 & $\leq 7.68$ & $100 \%$ \\
\hline$m i R-67 I-5 p$ & -0.42619047 & 0.018745 & $\leq 6.74$ & $100 \%$ \\
\hline $\mathrm{miR}-605$ & -0.21642858 & 0.018745 & $\leq 6.55$ & $100 \%$ \\
\hline miR-I224-5p & -0.52761906 & 0.018745 & $<6.84$ & $100 \%$ \\
\hline miR-202 & -0.632619 & 0.047273 & $\leq 6.94$ & $85 \%$ \\
\hline \multicolumn{5}{|c|}{ Cancer group2 } \\
\hline \multicolumn{5}{|c|}{ Over-expressed in current smokers } \\
\hline miR-934 & 0.65880954 & 0.02635 & $\geq 6.67$ & $100 \%$ \\
\hline $\mathrm{miR}-500$ & 0.4397619 & 0.063356 & $\geq 6.72$ & $92 \%$ \\
\hline $\mathrm{miR}-892 \mathrm{~b}$ & 0.41 & 0.171085 & $\geq 6.63$ & $92 \%$ \\
\hline \multicolumn{5}{|l|}{ AC } \\
\hline \multicolumn{5}{|c|}{ Over-expressed in asbestos-related samples } \\
\hline miR-199b-5p & 1.06 & 0.049309 & $\geq 7.46$ & $100 \%$ \\
\hline miR-374a & 0.894 & 0.057633 & $\geq 7.44$ & $100 \%$ \\
\hline let-7d & 1.416 & 0.067007 & $\geq 7.9$ & $100 \%$ \\
\hline let-7e & 1.28 & 0.067007 & $\geq 7.71$ & $100 \%$ \\
\hline miR-33I-3p & 1.208 & 0.067007 & $\geq 8.06$ & $100 \%$ \\
\hline miR-96 & 1.706 & 0.067007 & $\geq 7.54$ & $100 \%$ \\
\hline miR-24-I* & 0.314 & 0.067007 & $\geq 6.65$ & $100 \%$ \\
\hline \multicolumn{5}{|c|}{ Under-expressed in asbestos-related samples } \\
\hline miR-202 & -1.142 & 0.049309 & $\leq 6.94$ & $100 \%$ \\
\hline
\end{tabular}

a Mean log2 fold change between asbestos-related and nonrelated samples OR between ex-smokers and current smokers.

bLevel of expression associated with asbestos exposure or current smoking.

target genes were identified. The smoking-statusrelated results are hence not discussed further.

Since cancer Group 1 contained significantly more ACs compared with cancer Group 2, the differences between all asbestos-related and nonrelated $\mathrm{AC}$ samples (five exposed + five non- exposed) were analyzed. Eight significantly differentially expressed miRNAs (FDR $<0.07$ ) were able to classify all of the samples correctly (Fig. 3b, Table 3). Three of the miRNAs were the same as those identified in cancer Group 1 (Table 3). Integrated analysis with preprocessed 
TABLE 4. Asbestos-Related Inverse Correlation Between miR-I48b and its Target Genes in Cancer Group I, and Between miR-96 and let-7d/e and their Target Genes in AC Samples

\begin{tabular}{|c|c|c|c|}
\hline miRNA & $\begin{array}{l}\text { miRNA } \log _{2} \\
\text { fold change }\end{array}$ & $\begin{array}{l}\text { Target } \\
\text { gene }\end{array}$ & $\begin{array}{l}\text { Gene } \log _{2} \\
\text { fold change }\end{array}$ \\
\hline \multicolumn{4}{|c|}{ Cancer group I } \\
\hline \multirow[t]{3}{*}{ miR-I48b } & 0.265 & FOSB & -1.02 \\
\hline & & GADD45A & $-0.5 \mathrm{II}$ \\
\hline & & LTBPI & -0.122 \\
\hline \multicolumn{4}{|l|}{$A C$} \\
\hline \multirow[t]{15}{*}{$m i R-96$} & 2.21 & NCALD & $-1.27 \mid$ \\
\hline & & CACNA2D2 & -0.967 \\
\hline & & MTSSI & -0.88 \\
\hline & & EPB4 IL3 & -0.591 \\
\hline & & TAOKI & -0.529 \\
\hline & & $A K R / C I$ & -0.52 \\
\hline & & SLCIAI & -0.469 \\
\hline & & PLAGLI & -0.392 \\
\hline & & $P C$ & -0.317 \\
\hline & & HBEGF & -0.235 \\
\hline & & $\mathrm{FI} 3 \mathrm{AI}$ & -0.185 \\
\hline & & MYOID & -0.18 \\
\hline & & CYP2B7PI & -0.080 \\
\hline & & NDRGI & -0.056 \\
\hline & & SLC35AI & -0.058 \\
\hline let-7e & 1.13 & VAV3 & -0.503 \\
\hline \multirow[t]{2}{*}{ let-7d/e } & $1.37 / 1.13$ & IGF2BP3 & -2.772 \\
\hline & & $A P I S I$ & -0.207 \\
\hline
\end{tabular}

${ }^{\mathrm{a}}$ Median $\log _{2}$ fold change between asbestos-related and nonrelated samples.

mRNA data revealed 18 down-regulated target genes correlating with the over-expression of let7e/d or miR-96 (Table 4).

During preprocessing of the available aCGH data, it was noted that three of four asbestosrelated and none of four nonrelated AC samples displayed a loss at 10q26 (data not shown). This locus contains miR-202, which was found to be under-expressed in asbestos-related samples of both cancer Group 1 and the ACs (Table 3).

The samples from patients with SCG were also tested for asbestos-related changes and a significant over-expression of miR-205 was identified in the normal samples of exposed patients as compared to those from the nonexposed patients $\left(\right.$ FDR $<0.15, \log _{2}$ fold change $=0.71 ;$ predictive $\log _{2}$ expression level $\geq 7.02$, predictive value $100 \%$ ). Integrated analysis with preprocessed mRNA data revealed 23 down-regulated target genes (Supporting Information 9).

\section{DISCUSSION}

The main aim of this study was to reveal novel miRNAs deregulated in lung cancer and particularly in association with the patients' earlier asbestos exposure. Furthermore, miRNA expression data were integrated with mRNA and aCGH data from the same patients (Table 1). The miRNA expression profile differentiating tumor samples from their normal counterparts classified them into three distinct groups; one normal sample group and two tumor sample groups (Fig. 1, Table 2). The two tumor groups (cancer Groups 1 and 2) were treated separately in subsequent analyses and asbestos-related miRNAs were identified (Table 3, Fig. 3a). Integrated analysis also revealed inversely correlated target genes. In addition, several newly identified lung cancer associated miRNAs and target genes as well as correlating CNA were identified. Worth mentioning are the findings of the down-regulated target gene DOK4 in correlation with the well known SCC-specific miR-205 and the possible AC-specificity of miR-27a. The most significant findings are discussed below and all of the results can be viewed in the Supporting Information.

\section{Asbestos-Related miRNAs and Target Genes in Lung AC}

In cancer group 1 (Table 2), which was dominated by AC, 8 miRNAs were identified to be differentially expressed between asbestos-related and nonrelated samples. Three target genes, GADD45A, LTBP1 and FOSB, were found to be down-regulated in correlation with the overexpression of miR-148b (Table 4). GADD45A is a member of the growth arrest and DNA-damage inducible genes, which have been implicated as tumor suppressors (TS) in several human cancers (Zerbini and Libermann, 2005). Also FOSB has been shown to be down-regulated in certain cancers (Kim et al., 2010).

The AC samples were also analyzed separately for asbestos-related changes in miRNA expression; 8 miRNAs were detected, of which three were the same as those in the analysis on cancer group 1 (Table 3, Fig. 3b). One of these three, miR-202, has been shown to be down-regulated by 4-hydroxynoneal (HNE) in HL-60 leukemia cells (Pizzimenti et al., 2009). HNE exerts oxidative stress, which is also closely associated with asbestos-related effects (Nymark et al., 2008; Poli et al., 2008). This may indicate that underexpression of miR-202 is associated with asbestos-induced oxidative stress. Oxidative stress is also closely associated with chromosomal damage and, interestingly, the under-expression of $\mathrm{miR}$ 202 was correlated with a loss of its locus (10q26) 
in 3 of 4 asbestos-related AC samples, from which aCGH results were available. In contrast, none of the 4 nonrelated AC samples showed such a loss (data not shown). Loss of $10 \mathrm{q} 26$ has previously been implicated in lung cancer (Levin et al., 1994).

Although usually under-expressed in cancer, let-7d and $-7 \mathrm{e}$ were found to be over-expressed in asbestos-related ACs. Interestingly, overexpression of let-7 family members has also been reported in malignant mesothelioma (MM), where it has been postulated to be related to IL6-induced survival (Guled et al., 2009; Busacca et al., 2010).

miR-96 was also found to be over-expressed in the asbestos-related ACs. It has previously been shown to be highly expressed in several types of cancer (Navon et al., 2009). Two calcium-related genes $N C A L D$ (neurocalcin delta gene) and $C A C$ NA2D2 (calcium channel, voltage dependent, alpha 2/delta subunit 2) were found to be downregulated in correlation with the over-expression of miR-96 (Table 4). This may be interesting, since asbestos-induced cytotoxicity has been shown to involve modulation of intracellular calcium concentrations (Shukla et al., 2003). Furthermore, CACNA2D2 has been proposed to be a TS gene that links $\mathrm{Ca}^{2+}$ signaling with the pathogenesis of lung cancer (Carboni et al., 2003; Navon et al., 2009). It is located at the $3 \mathrm{p} 21.31$ TS cluster, which has been shown to be lost in association with asbestos exposure in lung cancer (Marsit et al., 2004; Nymark et al., 2006). $N C A L D$, a member of the visinin-like subfamily of neural $\mathrm{Ca}^{2+}$ sensor proteins (VSNL), has also been suggested to act as a TS (Braunewell and Szanto, 2009). Additionally, two other potentially interesting down-regulated miR-96 target genes were identified, namely MTSS1 and EPB41L3 (Table 4). Down-regulation of MTSS1 has been associated with a poor outcome in breast cancer and with nodal metastasis in gastric cancer, while EPB41L3 has been identified as a TS in ovarian cancer (Parr and Jiang, 2009; Dafou et al., 2010; Liu et al., 2010a).

\section{Lung Cancer Associated miRNAs and Target Genes, and Their Potential Role in Asbestos- Exposed Patients}

In the initial comparison between all of the tumor and corresponding normal samples, several previously known lung cancer-related miRNAs were identified and nine were newly discovered
(Fig. 1, Supporting Information 1; Yanaihara et al., 2006; Navon et al., 2009; Wang et al., 2009; Wu et al., 2009; Dacic et al., 2010; Gao et al., 2010; Liu et al., 2010b). In addition, inverse correlation of several target genes was detected (Supporting Information 4 and 5). For example, up-regulation of MAP4K4,KRAS, and SOX4 correlated with the under-expression of miR-145, miR-30d/181a, and miR-30d, respectively (Supporting Information 5) and down-regulation of DLC1 and PDZD2 correlated with the overexpression of $\mathrm{miR}-19 \mathrm{a}$ and $\mathrm{miR}-21 / 25$, respectively (Supporting Information 4). MAP4K4 has been shown to be up-regulated in many tumor cell lines and tissues, including lung cancer, and $K R A S$ is one of the most frequently mutated proto-oncogenes in lung cancer (Husgafvel-Pursiainen et al., 1999; Collins et al., 2006; Suda et al., 2010). SOX4 has been shown to act as an oncogene in several cancers and may be the target gene of a frequently found amplification at $6 p$ in lung cancer (Andersen et al., 2009; Medina et al., 2009). DLC1 (deleted in liver cancer 1) is frequently deleted in several solid cancers and PDZD2 has been shown to possess TS properties (Tam et al., 2008).

Four miRNAs were found to be affected by CNA in correlation with the deregulation in the tumor samples. In particular, miR-141 and miR200c showed a significant degree of correlation between over-expression and copy number gains at their locus (12p13.31). As far as we are aware, such a correlation has never been observed before, although the two events have been reported separately in lung cancer (Rydzanicz et al., 2008; Wu et al., 2009).

The identification of two separate cancer groups in the initial analysis on all samples naturally led to further analyses on the differences between the groups and they were found to be almost completely classifiable according to the expression of two miRNAs (miR-27a and miR939). Their deregulation was also correlated with CNA in the aCGH data (data not shown). mir27a was found to be over-expressed and affected by CNA more frequently in the $\mathrm{AC}$ dominated cancer group 1, while miR-939 was more frequently over-expressed and affected by CNA in cancer Group 2. This difference between the two cancer groups seemed to be useful in the identification of asbestos-related lung cancers. The eight asbestos-related miRNAs in cancer Group 1, in combination with miR-27a were able to separate (i) cancer Groups 1 and 2, and (ii) asbestos- 


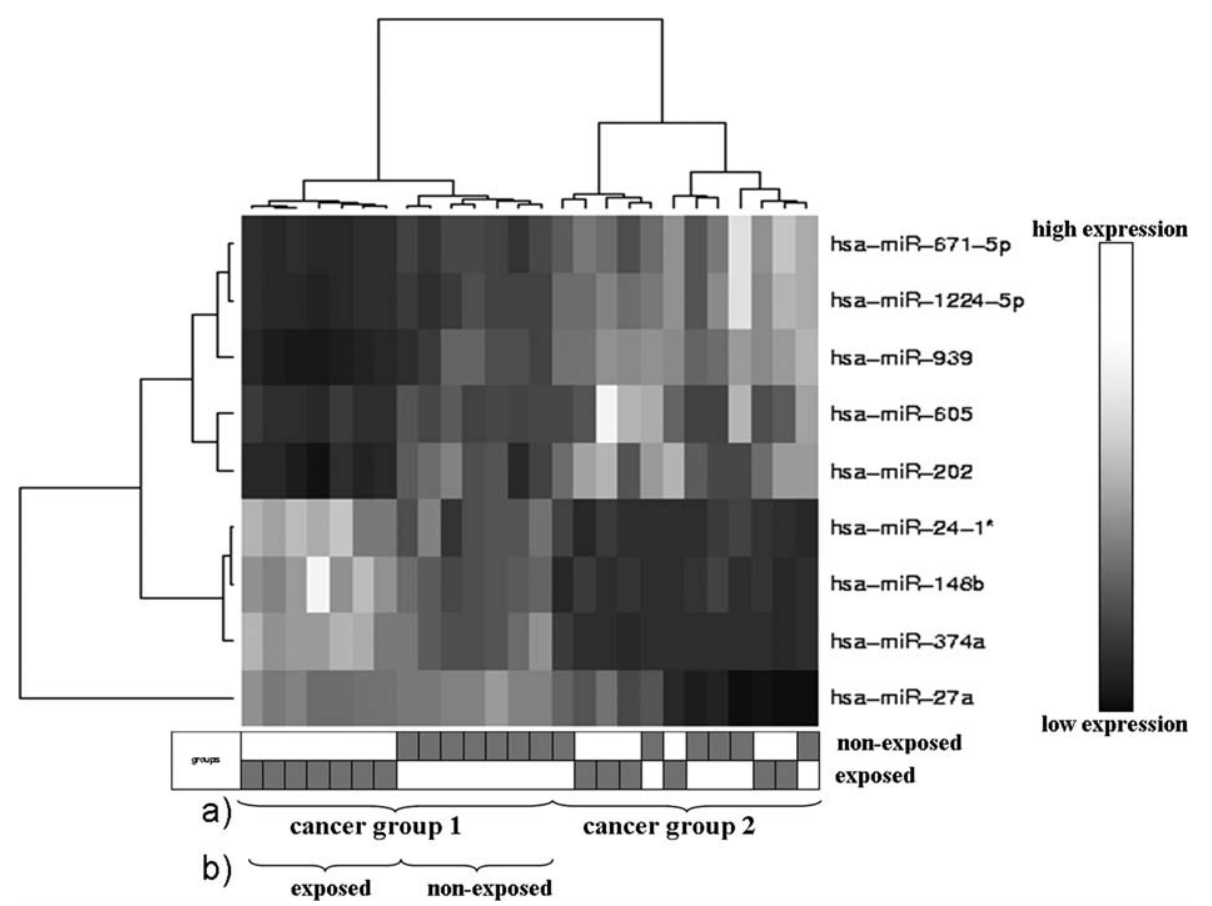

Figure 4. Classification capability of the eight asbestos-related miRNAs in cancer Group I and the cancer Group I-related miR-27a, (a) cancer Groups I and 2, and (b) asbestos-related and nonrelated tumor samples within cancer Group I.

related and nonrelated tumor samples within cancer Group 1 (Fig. 4).

Over-expression of miR-27a has been reported in gastric adenocarcinoma by Liu et al. (2009), and cancer group 1, which showed over-expression of miR-27a, was dominated by AC samples. This may indicate that mir-27a could be specific for AC histology. Indeed, mir-27a is located at 19 13.1, which has been reported to show frequent allelic imbalance (AI) in AC tumors (Sanchez-Cespedes et al., 2001; Ruosaari et al., 2008). In correlation with the over-expression of miR27a, 15 target genes were found to be down-regulated (Supporting Information 3). The calcium sensor SYT1 was the most strongly down-regulated (Fig. 2a) and since asbestos exposure is associated with the modulation of intracellular calcium levels, it is intriguing to speculate that the down-regulation of this calcium sensor gene in cancer Group 1 could confer sensitivity to the effects of asbestos and possibly increase the cells susceptibility to carcinogenesis (Shukla et al., 2003). Another down-regulated miR-27a target gene was $G C L C$, which is involved in glutathione synthesis. Interestingly, null phenotype polymorphisms in glutathione synthesizing genes have in some studies been proposed to increase the risk of MM as well as other asbestos-related diseases (Neri et al., 2008). Thus, down-regulation of
GCLC through miR-27a targeting may also sensitize the cells to the effects of asbestos.

\section{miR-205 in SCC and Its Potential Role in Asbestos-Exposed Patients}

A comparison between AC and SCG samples identified the highly SCG-specific miR-205. Several previous studies have reported the SCCspecificity of this miRNA; however, no clear target genes have been identified (Yanaihara et al., 2006; Lebanony et al., 2009; Bishop et al., 2010). miR-205 has also been shown to be highly expressed in cell lines of head and neck SCC and in esophageal SCG, pointing to a role in determining the squamous cell phenotype (Lebanony et al., 2009; Matsushima et al., 2010). Here, the over-expression of miR-205 correlated with the down-regulation of the target gene DOK4 (Fig. 2b). DOK4, an insulin receptor substrate (IRS5) gene, plays a role in cellular growth, signaling, and survival and may be involved in the MAP kinase pathway. In a recent study, DOK4/IRS5 was found to be both up- and down-regulated in NSCLC, with experiments pointing toward an epigenetic mechanism (Gray et al., 2008). This study indicates that the down-regulation in SCC may at least partly be due to miRNA targeting. Down-regulation of another insulin 
receptor substrate gene, IRS1, has been associated particularly with SCC in lung cancer (Han et al., 2006).

Furthermore, over-expression of mir-205 was also detected in the normal samples from asbestos-exposed patients with SCC compared with those from nonexposed patients. It is tempting to hypothesize that asbestos is able to induce the expression of miR-205 at an earlier stage of carcinogenesis in exposed individuals as compared with nonexposed subjects. Thus, although this miRNA is not specific for asbestos exposure in lung cancer, it may be an asbestos-specific early event in SCG. The over-expression of miR-205 in the asbestos-related samples correlated with the down-regulation of distinct target genes; e.g., TXNRD1, which is involved in oxidative stress; $S M A D 1$, which shows reduced protein levels in asbestos-treated mouse lungs and the histone demethylation involved gene $K D M 4 B$ at $19 \mathrm{p} 13.3$ (Supporting Information 9; Myllärniemi et al., 2008). AI and copy number loss at 19 p13.3 has been associated with asbestos exposure in nonAC histological types of lung cancer (Wikman et al., 2007; Ruosaari et al., 2008).

\section{miRNAs and Target Genes Potentially Associated with Early Stage Carcinogenesis of the Lung}

A much broader difference in miRNA expression was seen between normal lung from cancer patients and control samples (100 differentially expressed miRNAs) than between tumor and corresponding normal samples (34 differentially expressed miRNAs). A similar phenomenon could not be observed in the mRNA data, supporting the notion that miRNAs are more accurate and sensitive in classifying tissues according to origin, type, and possibly also to malignancy stage as compared to mRNA data (Lu et al., 2005). Seventy-seven percent of the 30 most significantly differentially expressed miRNAs were underexpressed in normal compared with control samples (Supporting Information 6). A similar deregulation of the whole miRNA biogenesis pathway in the early morphological modifications during cancer development has been postulated in a previous experimental study on squamous cell carcinogenesis (Mascaux et al., 2009). The study pointed to a two-step evolution for genome-wide miRNAs, first decreasing in abundance during early morphological modifications of bronchial epithelium and thereafter increasing in the later stages of lung carcinogenesis.
Among the under-expressed miRNAs were several members of the let-7 family (a, d, e, f, g, and i) which were not identified in the comparison between cancer and normal samples, although these vital miRNAs have been reported to play an important role in the development of lung cancer (Yanaihara et al., 2006; Wang et al., 2009). Consistent with our findings, Inamura et al. (2007) reported that let-7 under-expression occurred early during lung carcinogenesis.

In correlation with some of the underexpressed miRNAs, up-regulation of several target genes commonly known as TS genes was identified. Some examples are the proapoptotic genes EGR2 and EGR3, and the tumorigenesis limiting gene SOCS2 (Supporting Information 7). This may be evidence of an initial attempt to inhibit the carcinogenic events in the normal tissue surrounding the tumor. However, up-regulation of potential oncogenes were also identified, such as the leukemia associated gene MCL1, the inflammation involved gene PTGS2 (COX2) as well as the lung cancer associated genes NR4A2 and $C F H$. Inhibition of $N R 4 A 2$ has been shown to be associated with growth suppression in LKB1 (19p13) null lung tumors and $C F H$ auto-antibodies are associated with early stage NSCLC (Amornsiripanitch et al., 2010; Komiya et al., 2010).

\section{CONCLUSIONS}

Novel asbestos- and histology-associated miRNAs were identified. In addition, inverse correlation of specific target genes was discovered using integration analysis on miRNA and mRNA data from the same patient samples. CNA were also correlated with some of the deregulated miRNAs. The most significant observations strongly suggest a role for these miRNAs in either asbestos-related lung cancer or lung cancer with SCG histology, especially since the predictive accuracy is $100 \%$ in most cases (Table 3). Nevertheless, independent experiments are needed to confirm the findings. From a diagnostic perspective, these findings could potentially have important implications, many of which warrant further investigation.

\section{ACKNOWLEDGMENTS}

The authors thank Päivi Tuominen for the extraction of RNA, Antti Karjalainen for the interview of the patients, Harriet Wikman for the original planning of the sample group used in the 
study, Esa Vanhala for the fiber count analyses and Massimiliano Gentile for quick and efficient help with Chipster.

\section{REFERENCES}

Amornsiripanitch N, Hong S, Campa M, Frank M, Gottlin E, Patz EJ. 2010. Complement factor $\mathrm{H}$ autoantibodies are associated with early stage NSCLC. Clin Cancer Res 16:3226-3231.

Andersen CL, Christensen LL, Thorsen K, Schepeler T, Sorensen FB, Verspaget HW, Simon R, Kruhoffer M, Aaltonen LA, Laurberg S, Orntoft TF. 2009. Dysregulation of the transcription factors SOX4, CBFB and SMARCC1 correlates with outcome of colorectal cancer. Cancer Res 100:511-523.

Bishop J, Benjamin H, Cholakh H, Chajut A, Clark D, Westra W. 2010. Accurate classification of non-small cell lung carcinoma using a novel microRNA-based approach. Clin Cancer Res 16:610-619.

Braunewell K-H, Szanto A. 2009. Visinin-like proteins (VSNLs): interaction partners and emerging functions in signal transduction of a subfamily of neuronal $\mathrm{Ca} 2+$-sensor proteins. Cell Tiss Res 335:301-316.

Busacca S, Germano S, De Cecco L, Rinaldi M, Comoglio F, Favero F, Murer B, Mutti L, Pierotti M, Gaudino G. 2010. MicroRNA signature of malignant mesothelioma with potential diagnostic and prognostic implications. Am J Resp Cell Mol Biol 42:312-319.

Carboni GL, Gao B, Nishizaki M, Xu K, Minna JD, Roth JA, Ji L. 2003. CACNA2D2-mediated apoptosis in NSCLC cells is associated with alterations of the intracellular calcium signaling and disruption of mitochondria membrane integrity. Oncogene 22:615-626.

Churg A. 1997. Mineral analysis of the lung parenchyma. In: Crystal RG WJWE, Barnes PJ, editors. The Lung: Scientific Foundations. Philadelphia: Lippincott-Raven Pub. pp 2325-2338.

Collins CS, Hong J, Sapinoso L, Zhou Y, Liu Z, Micklash K, Schultz PG, Hampton GM. 2006. A small interfering RNA screen for modulators of tumor cell motility identifies MAP4K4 as a promigratory kinase. Proc Natl Sci USA 103:3775-3780.

Consensus Report. 1997. Asbestos, asbestosis, and cancer: The Helsinki criteria for diagnosis and attribution. Scand J Work Environ Health 23:311-316.

Dacic S, Kelly L, Shuai Y, Nikiforova MN. 2010. miRNA expression profiling of lung adenocarcinomas: Correlation with mutational status. Mod Pathol 23:1577-1582.

Dafou D, Grun B, Sinclair J, Lawrenson K, Benjamin E, Hogdall E, Kruger-Kjaer S, Christensen L, Sowter H, Al-Attar A, Edmondson R, Darby S, Berchuck A, Laird PW, Pearce CL, Ramus SJ, Jacobs IJ, Gayther SA. 2010. Microcell-mediated chromosome transfer identifies EPB41L3 as a functional suppressor of epithelial ovarian cancers. Neoplasia 2:579-589.

Gao W, Yu Y, Cao H, Shen H, Li X, Pan S, Shu Y. Deregulated expression of miR-21, miR-143 and miR-181a in non small cell lung cancer is related to clinicopathologic characteristics or patient prognosis. Biomed Pharmacother 64:399-408.

Gray S, Al-Sarraf N, Baird A, Gately K, McGovern E, O’Byrne K. 2008. Transcriptional regulation of IRS5/DOK4 expression in non-small-cell lung cancer cells. Clin Lung Cancer 9:367-374.

Guled M, Lahti L, Lindholm PM, Salmenkivi K, Bagwan I, Nicholson AG, Knuutila S. 2009. CDKN2A, NF2, and JUN are dysregulated among other genes by miRNAs in malignant mesotheliomaâ€"A miRNA microarray analysis. Genes Chromosomes Cancer 48:615-623.

Han C, Cho J, Moon J, Kim H, Kim S, Shin D, Chang J, Ahn C, Kim S, Chang Y. 2006. Clinical significance of insulin receptor substrate-I down-regulation in non-small cell lung cancer. Oncol Rep 16:1205-1210.

Husgafvel-Pursiainen K, Karjalainen A, Kannio A, Anttila S, Partanen T, Ojajärvi A, Vainio H. 1999. Lung cancer and past occupational exposure to asbestos. Role of p53 and K-ras mutations. Am J Respir Cell Mol Biol 20:667-674.

Inamura K, Togashi Y, Nomura K, Ninomiya H, Hiramatsu M, Satoh Y, Okumura S, Nakagawa K, Ishikawa Y. 2007. let-7 microRNA expression is reduced in bronchioloalveolar carcinoma, a non-invasive carcinoma, and is not correlated with prognosis. Lung Cancer 58:392-396.
Karjalainen A, Anttila S, Heikkila L, Karhunen P, Vainio H. 1993. Asbestos exposure among Finnish lung cancer patients: Occupational history and fiber concentration in lung tissue. Am J Ind Med 23:461-471.

Karjalainen A, Anttila S, Vanhala E, Vainio H. 1994. Asbestos exposure and the risk of lung cancer in a general urban population. Scand J Work Environ Health 20:243-250.

Kettunen E, Aavikko M, Nymark P, Ruosaari S, Wikman H, Vanhala E, Salmenkivi K, Pirinen R, Karjalainen A, Kuosma E, Anttila, S. 2009. DNA copy number loss and allelic imbalance at 2 p16 in lung cancer associated with asbestos exposure. $\mathrm{Br} \mathrm{J}$ Cancer 100:1336-1342.

Kim J, Lee J, Lee K, Lee J, Lee K, Jang K, Heo J, Choi S, Rhee J. 2010. RGS16 and FosB underexpressed in pancreatic cancer with lymph node metastasis promote tumor progression. Tumour Biol 31:541-548.

Komiya T, Coxon A, Park Y, Chen W-D, Zajac-Kaye M, Meltzer P, Karpova T, Kaye FJ. 2010. Enhanced activity of the CREB co-activator Crtc1 in LKB1 null lung cancer. Oncogene 29: 1672-1680.

LaDou J. 2004. The asbestos cancer epidemic. Environ Health Perspect 112:285-290.

Lahti L, Myllykangas S, Knuutila S, Kaski S. 2009. Dependency detection with similarity constraints. In: Adali T,Chanussot J,Jutten C,Larsen J, editors. Proceedings of the 2009 IEEE International Workshop on Machine Learning for Signal Processing XIX; Piscataway, NJ. pp.89-94.

Lebanony D, Benjamin H, Gilad S, Ezagouri M, Dov A, Ashkenazi K, Gefen N, Izraeli S, Rechavi G, Pass H, Nonaka D, Li J, Spector Y, Rosenfeld N, Chajut A, Cohen D, Aharonov R, Mansukhani M. 2009. Diagnostic assay based on hsa-miR-205 expression distinguishes squamous from nonsquamous non-small-cell lung carcinoma. J Clin Oncol 27:20302037

Levin N, Brzoska P, Gupta N, Minna J, Gray J, Christman M. 1994. Identification of frequent novel genetic alterations in small cell lung carcinoma. Cancer Res 54:5086-5091.

Liu K, Wang G, Ding H, Chen Y, Yu G, Wang J. 2010. Downregulation of metastasis suppressor 1(MTSS1) is associated with nodal metastasis and poor outcome in Chinese patients with gastric cancer. BMC Cancer 10:428a.

Liu T, Tang H, Lang Y, Liu M, Li X. 2009. MicroRNA-27a functions as an oncogene in gastric adenocarcinoma by targeting prohibitin. Cancer Lett 273:233-242.

Liu X, Sempere LF, Ouyang H, Memoli VA, Andrew AS, Luo Y, Demidenko E, Korc M, Shi W, Preis M, Dragnev KH, Li H, DiRenzo J, Bak M, Freemantle SJ, Kauppinen S, Dmitrovsky E. 2010. MicroRNA-31 functions as an oncogenic microRNA in mouse and human lung cancer cells by repressing specific tumor suppressors. J Clin Invest 120:1298-1309b.

Lopez-Romero P, Gonzalez M, Callejas S, Dopazo A, Irizarry R. 2010. Processing of Agilent microRNA array data. BMC Res Notes 3:18.

Lu J, Getz G, Miska EA, Alvarez-Saavedra E, Lamb J, Peck D, Sweet-Cordero A Ebert BL Mak RH, Ferrando AA, Downing JR, Jacks T, Horvitz HR, Golub TR. 2005. MicroRNA expression profiles classify human cancers. Nature 435:834-838.

Marsit CJ, Hasegawa M, Hirao T, Kim D-H, Aldape K, Hinds PW, Wiencke JK, Nelson HH, Kelsey KT. 2004. Loss of heterozygosity of chromosome 3 p21 is associated with mutant TP53 and better patient survival in non-small-cell lung cancer. Cancer Res 64:8702-8707.

Mascaux C, Laes JF, Anthoine G, Haller A, Ninane V, Burny A, Sculier JP. 2009. Evolution of microRNA expression during human bronchial squamous carcinogenesis. Eur Respir J 33: 352-359.

Matsushima K, Isomoto H, Kohno S, Nakao K. 2010. MicroRNAs and esophageal squamous cell carcinoma. Digestion 82:138144.

Medina P, Castillo S, Blanco S, Sanz-Garcia M, Largo C, Alvarez S, Yokota J, Gonzalez-Neira A, Benitez J, Clevers H, Cigudosa JC, Lazo PA, Sanchez- Cespedes M. 2009. The SRY-HMG box gene, $\mathrm{SOX} 4$, is a target of gene amplification at chromosome 6p in lung cancer. Hum Mol Gen 18:1343-1352.

Myllarniemi M, Lindholm P, Ryynanen MJ, Kliment CR, Salmenkivi K, Keski-Oja J, Kinnula VL, Oury TD, Koli K. 2008. Gremlin-mediated decrease in bone morphogenetic protein signaling promotes pulmonary fibrosis. Am J Respir Crit Care Med 177:321-329. 
Navon R, Wang H, Steinfeld I, Tsalenko A, Ben-Dor A, Yakhini Z. 2009. Novel rank-based statistical methods reveal MicroRNAs with differential expression in multiple cancer types. PLoS ONE 4:e8003.

Neri M, Ugolini D, Dianzani I, Gemignani F, Landi S, Cesario A, Magnani C, Mutti L, Puntoni R, Bonassi S. 2008. Genetic susceptibility to malignant pleural mesothelioma and other asbestos-associated diseases. Mutat Res/Rev Mutat Res/Proc 5th Int Conf Environ Mutagens Hum Populations (ICEMHP) 659:126136.

Nymark P, Wikman H, Ruosaari S, Hollmen J, Vanhala E, Karjalainen A, Anttila S, Knuutila S. 2006. Identification of specific gene copy number changes in asbestos-related lung cancer. Cancer Res 66:5737-5743.

Nymark P, Wikman H, Hienonen-Kempas T, Anttila S. 2008. Molecular and genetic changes in asbestos-related lung cancer. Cancer Lett 265:1-15.

Nymark P, Kettunen E, Aavikko M, Ruosaari S, Kuosma E, Vanhala E, Salmenkivi K, Pirinen R, Karjalainen A, Knuutila S, Wikman H, Anttila S. 2009. Molecular alterations at 9q33.1 and polyploidy in asbestos-related lung cancer. Clin Canc Res 15:468-475.

Parr C, Jiang WG. 2009. Metastasis suppressor 1 (MTSS1) demonstrates prognostic value and anti-metastatic properties in breast cancer. Eur J Cancer 45:1673-1683.

Pizzimenti S, Ferracin M, Sabbioni S, Toaldo C, Pettazzoni P, Dianzani MU, Negrini M, Barrera G. 2009. MicroRNA expression changes during human leukemic HL-60 cell differentiation induced by 4-hydroxynonenal, a product of lipid peroxidation. Free Radic Biol Med 46:282-288.

Poli G, Schaur R, Siems W, Leonarduzzi G. 2008. 4-Hydroxynonenal: A membrane lipid oxidation product of medicinal interest. Med Res Rev 28:569-631.

Pradervand S, Weber J, Thomas Jrm, Bueno M, Wirapati P, Lefort K, Dotto GP, Harshman K. 2009. Impact of normalization on miRNA microarray expression profiling. RNA 15:493501.

Ruosaari S, Nymark P, Aavikko M, Kettunen E, Knuutila S, Hollmen J, Norppa H, Anttila S. 2008. Aberrations of chromosome 19 in asbestos-associated lung cancer and in asbestos-induced micronuclei of bronchial epithelial cells in vitro. Carcinogenesis 29:913-917.

Rydzanicz M, Giefing M, Ziolkowski A, Kasprzyk M, Gabriel A, Dyszkiewicz W, Szyfter K. 2008. Nonrandom DNA copy number changes related to lymph node metastases in squamous cell carcinoma of the lung. Neoplasma 55:493500.

Sanchez-Cespedes M, Ahrendt SA, Piantadosi S, Rosell R, Monzo M, Wu L, Westra WH, Yang SC, Jen J, Sidransky D. 2001. Chromosomal Alterations in Lung Adenocarcinoma from Smokers and Nonsmokers. Cancer Res 61:1309_ 1313.

Shukla A, Ramos-Nino M, Mossman B. 2003. Cell signaling and transcription factor activation by asbestos in lung injury and disease. Int J Biochem. Cell Biol 35:1198-1209.

Suda K, Tomizawa K, Mitsudomi T. 2010. Biological and clinical significance of KRAS mutations in lung cancer: An oncogenic driver that contrasts with EGFR mutation. Cancer Metas Rev 29:49-60.

Tam CW, Liu VWS, Leung WY, Yao K-M, Shiu SYW. 2008. The autocrine human secreted PDZ domain-containing protein 2 (sPDZD2) induces senescence or quiescence of prostate, breast and liver cancer cells via transcriptional activation of p53. Cancer Lett 271:64-80.

Vainio H, Boffetta P. 1994. Mechanisms of the combined effect of asbestos and smoking in the etiology of lung cancer. Scand J Work Environ Health 20:235-242.

Wang Q, Xu W, Habib N, Xu R. 2009. Potential uses of microRNA in lung cancer diagnosis, prognosis, and therapy. Curr Cancer Drug Targ 9:572-594.

Venkatraman E, Olshen A. 2007. A faster circular binary segmentation algorithm for the analysis of array CGH data. Bioinformatics 23:657-663.

Wikman H, Ruosaari S, Nymark P, Sarhadi V, Saharinen J, Vanhala E, Karjalainen A, Hollmén J, Knuutila S, Anttila S. 2007. Gene expression and copy number profiling suggests the importance of allelic imbalance in 19p in asbestos-associated lung cancer. Oncogene 26:4730-4737.

Wu X, Piper-Hunter MG, Crawford M, Nuovo GJ, Marsh CB, Otterson GA, Nana-Sinkam SP. 2009. MicroRNAs in the pathogenesis of lung cancer. J Thorac Oncol 4:10281034 .

Yanaihara N, Caplen N, Bowman E, Seike M, Kumamoto K, Yi M, Stephens RM, Okamoto A, Yokota J, Tanaka T, Calin GA, Liu C-G, Croce CM, Harris CC. 2006. Unique microRNA molecular profiles in lung cancer diagnosis and prognosis. Cancer Cell 9:189-198.

Zerbini L, Libermann T. 2005. GADD45 deregulation in cancer: frequently methylated tumor suppressors and potential therapeutic targets. Clin Cancer Res 11:6409-6413. 